CLINICAL STUDY

\title{
The role of sonoelastography in acute, subacute and chronic thyroiditis: a novel application of the method
}

\author{
Marek Ruchala*, Ewelina Szczepanek-Parulska*, Ariadna Zybek, Jerzy Moczko ${ }^{1}$, Agata Czarnywojtek, \\ Grzegorz Kaminski ${ }^{2}$ and Jerzy Sowinski \\ Department of Endocrinology, Metabolism and Internal Medicine, Poznan University of Medical Sciences, 49 Przybyszewskiego Street, 60-355 Poznan, \\ Poland, ${ }^{1}$ Department of Computer Science and Statistics, 79 Dabrowskiego Street, $60-529$ Poznan, Poland and ${ }^{2}$ Department of Endocrinology and Isotope \\ Therapy, Military Institute of Medicine, 128 Szaserow Street, 04-141 Warsaw, Poland \\ (Correspondence should be addressed to M Ruchala; Email: mruchala@ump.edu.pl)
}

*(M Ruchala and E Szczepanek-Parulska contributed equally to this work)

\begin{abstract}
Objective: Reports on sonoelastography, which provide an objective estimation of tissue elasticity, are scarce in terms of thyroiditis. The aim of this study was to prospectively assess the applicability of sonoelastography in different types of thyroiditis.

Design: The study assessed and compared the thyroid tissue stiffness in patients with acute thyroiditis (AT), subacute thyroiditis (SAT), and chronic autoimmune thyroiditis (CAT) with healthy control subjects (CS), followed up for 10 weeks.

Methods: The study group consisted of two patients with AT, 18 patients with SAT, 18 patients with CAT, and 40 CS matched for age and gender. Sonoelastography was performed at baseline, at a 4-week follow-up during treatment, and at 10 weeks following diagnosis and treatment initiation.

Results: Thyroid tissue stiffness was higher in SAT at baseline $(214.26 \pm 32.5 \mathrm{kPa})$ in comparison with values recorded at a 4-week follow-up $(45.92 \pm 17.4 \mathrm{kPa})$ and at 10 weeks following diagnosis and treatment initiation $(21.65 \pm 5.3 \mathrm{kPa}, P<0.0001)$. Baseline thyroid stiffness in SAT was higher than that found in CAT $(36.15 \pm 18.7 \mathrm{kPa}, P<0.0001)$ and $\mathrm{CS}(16.18 \pm 5.4 \mathrm{kPa}, P<0.0001)$. In the remission of SAT, thyroid stiffness was lower than that found in CAT $(P=0.006)$, while it was higher than that in CS $(P=0.0008)$. No difference was observed between thyroid stiffness in SAT at 4-week follow-up and in CAT. Patients with CAT presented higher thyroid stiffness than CS $(P<0.0001)$, which was not influenced by L-thyroxine treatment. Thyroid stiffness in patients with AT was 216.6 and $241.9 \mathrm{kPa}$ at baseline; after treatment, it decreased to 17.93 and $85.348 \mathrm{kPa}$ respectively. Conclusions: Sonoelastography may assist in the diagnosis and treatment monitoring of AT, SAT and CAT, as well as in the differentiation of the various types of thyroiditis.
\end{abstract}

European Journal of Endocrinology 166 425-432

\section{Introduction}

Traditionally, palpation has been a part of a routine clinical evaluation of the thyroid, the aim of which is to examine tissue firmness. However, this method is highly subjective, and its interpretation varies depending on the size and location of the lesions, as well as the experience of the physician. Sonoelastography, sometimes referred to as 'electronic palpation', is a novel imaging technique used for the noninvasive and objective reconstruction of tissue stiffness, which measures the degree of its deformation in response to the application of an external force (1). It is based on the principle that abnormal tissue, affected with fibrosis, inflammatory infiltration or neoplastic process, tends to be stiffer than healthy tissue. The possibility of implementing this method in commercial ultrasound (US) systems and using it during routine US examinations is one of its great advantages. Although available from 1990, it was first used in thyroid examination in 2005 by Lyshchik et al. (2). Usefulness of the method in differentiating benign and malignant thyroid tumors has been widely described (3). Nevertheless, there is still no clear conclusion about its true clinical utility $(2,3,4)$. However, the sonoelastographic image of thyroid inflammatory diseases has only occasionally been the subject of investigation.

Although acute thyroiditis (AT), subacute thyroiditis (SAT), and chronic autoimmune thyroiditis (CAT) have dissimilar origin and require different management, these conditions may share some parallel clinical, biochemical, and sonographic features, which might lead to confusion and, as a result, diagnostic difficulties. On the other hand, thyroid sonoelastography was found to be exceptionally useful in the evaluation of thyroid nodules; nevertheless, it remains unknown whether the 
implementation of this method might bring further insight into the evaluation of thyroiditis. It is also uncertain whether a coexisting thyroid inflammation might influence the result of sonoelastographic evaluation of thyroid focal lesions. Thus, the aim of this study was to prospectively assess the sonoelastographic images of AT, SAT, and CAT at baseline and during remission, as well as to evaluate the applicability of sonoelastography in the diagnosis, differentiation, and treatment monitoring of the various types of thyroiditis.

\section{Subjects and methods}

\section{Subjects}

The studied group consisted of two patients with AT, 18 patients with granulomatous SAT, 18 patients with CAT and a group of 40 control subjects (CS) without thyroid pathology referred to the department for other conditions, matched for age and gender.

The patients underwent clinical examinations, routine blood tests, conventional US, followed by fine-needle aspiration biopsy (FNAB) if indicated, and a sonoelastographic examination. AT was diagnosed on the basis of clinical symptoms, including fever and painful goiter, accompanied by local lymphadenopathy, as well as the results of conventional US and cytological examination of the specimen obtained during FNAB. SAT was diagnosed in the presence of painful goiter, accompanied by an elevated erythrocyte sedimentation rate (ESR), moderately increased body temperature, decreased radioisotope uptake on the thyroid scintiscan, and a typical image acquired using conventional US. The latter involved an increase in the volume of the thyroid gland, especially in depth; it was accompanied by ill-defined regions of the thyroid parenchyma, characterized by areas of heterogeneous and decreased echogenicity, smoothly turning into those of normal echogenicity. To the group with CAT patients presenting hypothyroidism, elevated antithyroid autoantibodies and a diffusely decreased echogenicity of the thyroid parenchyma on conventional US were included. Subjects clinically and biochemically euthyroid, with a negative history of thyroid disease, and presenting no thyroid pathology on conventional thyroid US were classified as CS.

The protocol of the study was accepted by the local ethics committee, and all the participants gave informed consent to participate.

\section{Serum assays}

ESR after $1 \mathrm{~h}$ was calculated by a classical method, while concentration of C-reactive protein (CRP) was assessed by highly sensitive particle-enhanced immunoturbidimetric method. Assessment of hormone concentration was performed using Hitachi Cobas e601 chemiluminescent analyzer (Roche Diagnostics) and included measurement of serum TSH, free triiodothyronine $\left(\mathrm{FT}_{3}\right)$, and free thyroxine $\left(\mathrm{FT}_{4}\right)$. Thyroid autoantibody concentrations (TSH receptor antibody, TRAb; antithyroid peroxidase antibody, TPOAb; and antithyroglobulin antibody, $\mathrm{TgAb}$ ) were assessed by radioimmunological method using commercially available BRAMHS anti-TPO, anti-Tg, and TRAK RIA kits and scintillation gamma counter (LKB Wallac CliniGamma 1272).

\section{Thyroid imaging}

At baseline, all the patients underwent conventional US and sonoelastographic evaluation using an AIXPLORER system by Supersonic Imagine, with the use of Shear Wave Elastography. All the examinations were performed by two experienced thyroid sonographers (M R and E S) and the obtained results were averaged. The stiffness of thyroid parenchyma was assessed in both a qualitative and a quantitative manner. By measuring the propagation velocity of the shear waves at every point of the image, an elasticity map could be deduced. This included color-coded displays depicting tissue stiffness using a color scale from blue (soft) through green and yellow (medium elasticity) to red (hard). The classification of the affected thyroid parenchyma in a 4-point modified Ueno scale was used to describe tissue stiffness, where elasticity score (ES) I meant completely normal thyroid elasticity (blue color); ES II, parenchyma of predominantly normal elasticity, however, containing areas of slightly increased stiffness (green and yellow color); ES III, parenchyma of intermediate elasticity, where yellow color predominates and may contain some tiny areas of highly increased stiffness, depicted in red; and ES IV, most of the thyroid parenchyma presents highly increased stiffness, with predominating red color (5). The classical 5-point Ueno elasticity scale was modified, as regions affected with thyroid inflammation presenting no clear margins were evaluated. The quantitative information was depicted as a stiffness index and expressed in kilopascals on a continuous scale. Tissue stiffness was quantified by Young's Module E corresponding to the speed of propagation of the shear wave. As the method gives a possibility to evaluate stiffness at each point of interest, the stiffest area of a lesion was chosen for quantitative assessment of parenchymal firmness.

\section{Cytological examination}

US-guided FNAB of some detected lesions was performed to confirm the diagnosis and exclude thyroid malignancy. The cytological evaluation of the obtained specimens was performed by the two pathologists as a routine medical procedure in the Department of Pathology at Poznan University of Medical Sciences. 


\section{Interventions}

Patients diagnosed with AT were given a course of widespectrum antibiotics (third-generation cephalosporin) intravenously. All subjects diagnosed with SAT underwent an 8-week anti-inflammatory treatment with gradually decreasing doses of prednisone (from 40 to $5 \mathrm{mg}$ /day). Adjuvant treatment included $20 \mathrm{mg}$ omeprazole and supplementation with potassium chloride. In subjects diagnosed with CAT, $\mathrm{L}_{-} \mathrm{T}_{4}$ substitution was introduced to restore euthyroidism. All the patients underwent prospective observation, with sonographic and sonoelastographic assessment during a 4-week follow-up visit and at 10 weeks following diagnosis and treatment initiation.

\section{Statistical analysis}

First, the relevant parameters were compared at baseline, during a 4-week follow-up, and at 10 weeks following diagnosis and treatment initiation for both the SAT and CAT patients using a nonparametric Friedman test; subsequently, a multiple comparison analysis was carried out with the post hoc Dunn test. Next, the results were confirmed with the Quade test, due to the extremely low $P$ values. The exact $P$ value was estimated on the basis of permutational techniques using STATXACT-9 statistical software (Cytel Software Corporation, Cambridge, MA, USA).

Comparisons between patients with SAT and subjects diagnosed with CAT and CS were performed with the nonparametric Mann-Whitney $U$ test. As mentioned earlier, the exact $P$ values confirmed the decisions based on the standard asymptotical $P$ values. Patients with AT could not be involved in the statistical evaluation, since this group included very few subjects.

\section{Results}

The age and gender structure of the analyzed subgroups was as follows:

- patients diagnosed with AT - two women, aged 40 and 52 years
- patients diagnosed with SAT - 16 women and two men, aged 27-74 (median 49.5) years

- patients diagnosed with CAT - 16 women and two men, aged 25-71 (median 48) years

- CS - 36 women and four men, aged 21-78 (median $47.5)$ years.

FNAB was performed in 18 patients to confirm the diagnosis. In 13 of them, cytological diagnosis was consistent with SAT, in two with AT, and three biopsies were nondiagnostic. In the latter, SAT was diagnosed on the basis of clinical, laboratory, and sonographic data. Patients with CAT were diagnosed on the basis of clinical and laboratory data, and sonographic image; hence, they were not subject to FNAB.

A significant difference in thyroid tissue stiffness (qualitatively expressed on a 4-point scale, as well as quantitatively through the estimation of Young's Module E) was noted between SAT at baseline $(214.26 \pm 32.5 \mathrm{kPa})$ in comparison with values recorded at a 4 -week follow-up visit $(45.92 \pm 17.4 \mathrm{kPa})$ and at 10 weeks following diagnosis and treatment initiation $(21.65 \pm 5.3 \mathrm{kPa})$. Changes of the elastic properties of thyroid parenchyma were accompanied by a gradual normalization of biochemical parameters. The results of thyroid tissue stiffness and biochemical assessment of the patients, at the three phases of SAT, are presented in Table 1, together with the level of statistical significance. The stiffness of the thyroid parenchyma in SAT at baseline was additionally significantly higher when compared with that found in CAT $(36.15 \pm 18.7 \mathrm{kPa})$ or CS $(16.18 \pm 5.4 \mathrm{kPa})$, and with treatment was gradually restored to values close to normal (Fig. 1). Representative sonoelastographic images taken in SAT at baseline and during the recovery phase are presented in Fig. 2. The differences between thyroid tissue elasticity in the three phases of SAT, CAT and CS, assessed quantitatively as Young's Module E, are presented in Table 2. Table 3 presents thyroid tissue stiffness evaluated qualitatively on a 4-point scale, where I indicates the lowest stiffness and IV indicates the highest. At the 4-week follow-up, no significant difference was observed between thyroid tissue stiffness in SAT $(45.92 \pm 17.4 \mathrm{kPa})$ and CAT (36.15 $\pm 18.7 \mathrm{kPa})$. However, during remission, after

Table 1 Results of sonoelastographic and biochemical assessment of patients with SAT performed at baseline, on a 4-week follow-up, and at 10 weeks following diagnosis and treatment initiation.

\begin{tabular}{|c|c|c|c|c|}
\hline Parameters & $\begin{array}{c}\text { SAT } \\
\text { (baseline) (1) }\end{array}$ & $\begin{array}{c}\text { SAT } \\
\text { (4-week follow-up) (2) }\end{array}$ & $\begin{array}{c}\text { SAT } \\
\text { (10-week follow-up) (3) }\end{array}$ & $P$ value \\
\hline Young's Module E (kPa) & $214.26 \pm 32.5$ & $45.92 \pm 17.4$ & $21.65 \pm 5.3$ & $P<0.0001$ \\
\hline $\operatorname{ESR}(\mathrm{mm} / \mathrm{h})$ & $62.6 \pm 25.0$ & $16.2 \pm 7.9$ & $7.1 \pm 3.7$ & $P<0.0001$ \\
\hline CRP $(\mathrm{mg} / \mathrm{l})$ & $71.7 \pm 71.9$ & $10.2 \pm 12.9$ & $1.6 \pm 1.8$ & $P<0.0001$ \\
\hline TSH $(\mu \mathrm{IU} / \mathrm{ml})$ & $0.091 \pm 0.187$ & $3.864 \pm 3.950$ & $2.059 \pm 0.702$ & $\begin{array}{c}\text { (1) vs (2) } P<0.0001 \text { (1) vs (3) } \\
P=0.0039 \text { (2) vs (3) NS }\end{array}$ \\
\hline $\mathrm{FT}_{4}(\mathrm{pmol} / \mathrm{l})$ & $30.38 \pm 12.47$ & $14.53 \pm 2.88$ & $15.28 \pm 2.09$ & $\begin{array}{c}\text { (1) vs (2) } P<0.0001 \text { (1) vs (3) } \\
P=0.006 \text { (2) vs (3) NS }\end{array}$ \\
\hline
\end{tabular}

SAT, subacute thyroiditis; ESR, erythrocyte sedimentation rate; CRP, C-reactive protein; $\mathrm{FT}_{4}$, free thyroxine; NS, statistically nonsignificant. 


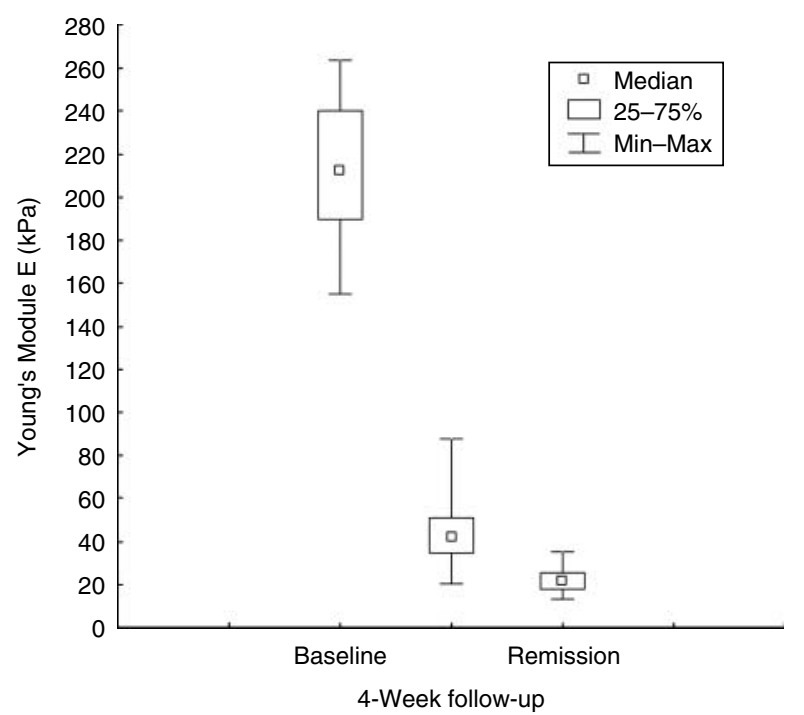

Figure 1 Young's Module $\mathrm{E}(\mathrm{kPa})$ reflecting the quantitative values of thyroid stiffness in the three phases of subacute thyroiditis (at baseline, on 4-week follow-up, and during remission).

treatment, patients with SAT presented significantly lower thyroid stiffness $(21.65 \pm 5.3 \mathrm{kPa})$ than that found in CAT $(36.15 \pm 18.7 \mathrm{kPa})$, while it was still higher in comparison with healthy CS (16.18 $\pm 5.4 \mathrm{kPa})$. Patients with CAT presented significantly higher thyroid stiffness in comparison with CS and thyroid elasticity was not influenced by $\mathrm{L}_{-} \mathrm{T}_{4}$ treatment.

Thyroid stiffness at baseline in the two women with AT was equal to 216.6 and $241.9 \mathrm{kPa}$ (IV) respectively, while during remission, after the evacuation of puss and with antibacterial treatment, it decreased to $17.93 \mathrm{kPa}$ (I) and $85.348 \mathrm{kPa}$ (II) respectively (Fig. 3). Moreover, in subjects with AT or SAT, the region of the thyroid presenting increased stiffness in the sonoelastographic examination corresponded directly to the area most painful on palpation.

\section{Discussion}

Sonoelastography combines the advantages of highfrequency US and the possibility to assess the lesion's stiffness (2). This novel sonographic technique is based on the difference in the viscoelastic properties between normal and pathological thyroid tissue, which corresponds to the tissue architecture. To date, sonoelastography has been mainly applied in the diagnostics of thyroid nodular disease (3). However, very little is known about the sonoelastographic image of inflammatory thyroid diseases.

A rare thyroid pathology clinically associated with an increased thyroid consistency is Riedel's thyroiditis (6). The sonoelastographic image of Riedel's thyroiditis in two patients has just been described, revealing heterogeneous stiffness of thyroid parenchyma ranging from 21 to $281 \mathrm{kPa}(7)$. Thyroid tissue stiffness was found to be significantly higher in Graves' disease, as well as in Hashimoto's thyroiditis, when compared with healthy controls (8). Recently, our team has described the sonoelastographic image of two patients with SAT, characterized by significantly increased thyroid stiffness at baseline, which restored to values close to normal with remission (9). However, a literature search reveals no reports on sonoelastographic examination in AT.

AT usually presents with signs of both local and systemic inflammation. The patient may have fever up to $38-40{ }^{\circ} \mathrm{C}$ with malaise. On palpation, the thyroid may appear enlarged and tender, with pain radiating to

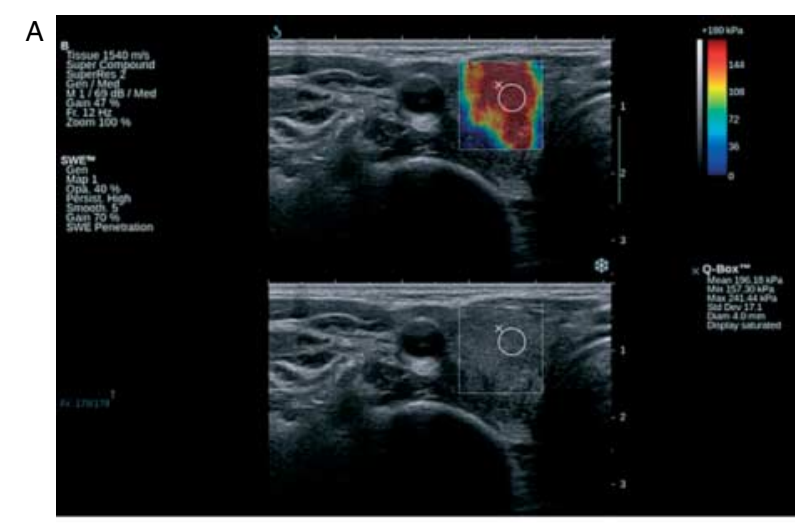

B

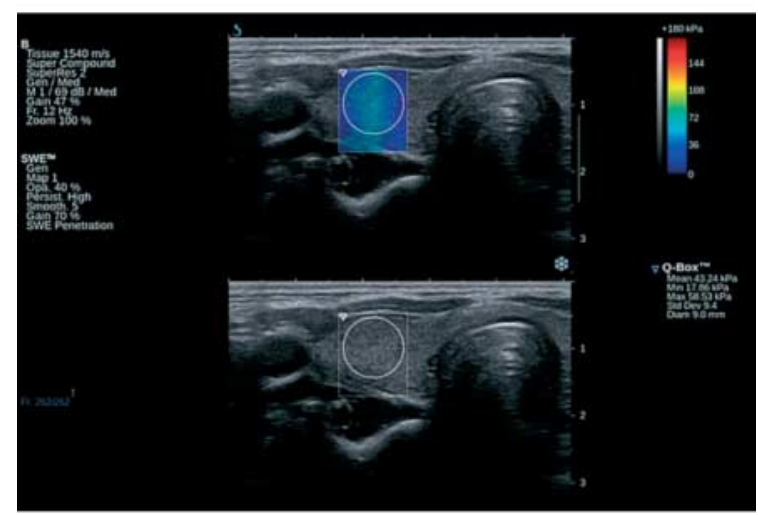

C

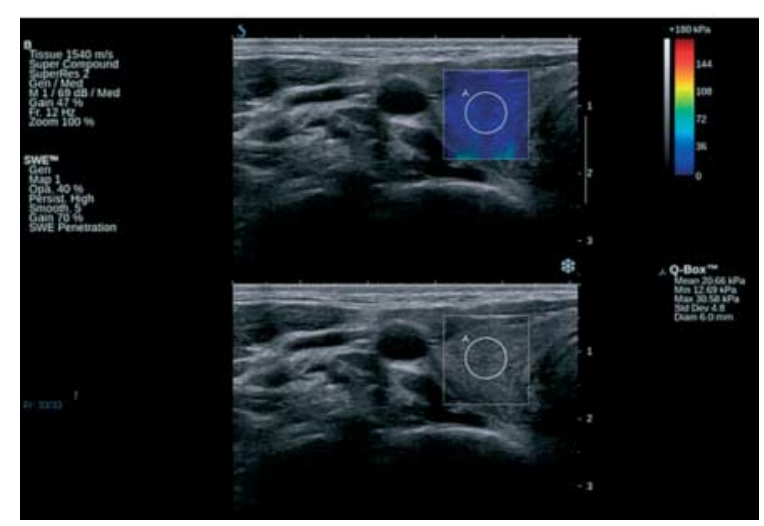

Figure 2 Thyroid stiffness assessed by sonoelastography in subacute thyroiditis (SAT) (A) at baseline, (B) on a 4-week follow-up, and $(C)$ during remission. 
Table 2 Thyroid tissue stiffness expressed in Young's Module E (kPa) in the three phases of SAT, compared with CAT and CS.

\begin{tabular}{|c|c|c|c|c|c|}
\hline \multirow[b]{2}{*}{ Young's Module E (kPa) } & \multicolumn{3}{|c|}{ SAT } & \multirow[b]{2}{*}{$\begin{array}{l}\text { CAT baseline } \\
(36.15 \pm 18.7)\end{array}$} & \multirow[b]{2}{*}{$\begin{array}{l}\text { CS baseline } \\
(16.18 \pm 5.4) \\
\end{array}$} \\
\hline & $\begin{array}{l}\text { Baseline } \\
(214.26 \pm 32.5)\end{array}$ & $\begin{array}{l}\text { 4-week follow-up } \\
(45.92 \pm 17.4)\end{array}$ & $\begin{array}{l}\text { 10-week follow-up } \\
(21.65 \pm 5.3)\end{array}$ & & \\
\hline \multicolumn{6}{|l|}{ SAT } \\
\hline Baseline $(214.26 \pm 32.5)$ & $\mathrm{X}$ & $P<0.0001$ & $P<0.0001$ & $P<0.0001$ & $P<0.0001$ \\
\hline 4-week follow-up $(45.92 \pm 17.4)$ & $P<0.0001$ & $\mathrm{x}$ & $P<0.0001$ & NS & $P<0.0001$ \\
\hline 10-week follow-up $(21.65 \pm 5.3)$ & $P<0.0001$ & $P<0.0001$ & $x$ & $P<0.006$ & $P<0.0008$ \\
\hline \multicolumn{6}{|l|}{ CAT } \\
\hline $\begin{array}{l}\text { Baseline }(36.15 \pm 18.7) \\
\text { CS }\end{array}$ & $P<0.0001$ & NS & $P<0.006$ & $\mathrm{x}$ & $P<0.0001$ \\
\hline Baseline $(16.18 \pm 5.4)$ & $P<0.0001$ & $P<0.0001$ & $P<0.0008$ & $P<0.0001$ & $\mathrm{X}$ \\
\hline
\end{tabular}

SAT, subacute thyroiditis; CAT, chronic autoimmune thyroiditis; CS, control subjects; kPa, kilo-Pascal; NS, statistically nonsignificant.

the mandible, ear or occiput. Biochemically, leukocytosis and moderately increased ESR and CRP, but not accompanied by disturbances in thyroid function, are detected (10). On the other hand, SAT may similarly manifest with increased body temperature, a significant elevation of ESR and CRP but rather no leukocytosis, firm and painful goiter, with pain of typical radiation. However, SAT is usually accompanied by thyroid dysfunction (11). CAT can be diagnosed at any thyroid functional state, but typically it is not characterized by painful thyroid enlargement and biochemical or clinical signs of inflammation. Nevertheless, some patients with CAT may complain of thyroid tenderness, which is accompanied by increased temperature and malaise, as well as moderately elevated ESR and CRP, and thus might be misdiagnosed as SAT (12). Though increased antithyroid autoantibodies are indicative of CAT, their presence does not exclude the diagnosis of other types of thyroiditis $(13,14)$.

The conventional sonographic image of AT and SAT might be very similar in the first phase of the disease and is characterized by ill-defined regions of heterogeneous decreased echogenicity $(15,16)$. However, later in AT, formation of an abscess can be observed, while in SAT, the echogenicity and thyroid size become normal during the recovery phase, with no residual changes. Though the typical image of SAT in conventional US seems clear, focal distortion and heterogeneity may only be found in some of the patients. In the study by Espinoza et al. (13), such an image presented in only eight out of 22 patients with SAT. On the other hand, CAT on conventional US is most often characterized by a diffusely decreased echogenicity, coupled with a varying extent of fibrosis (12).

On Doppler examination, in SAT, a near absence of vascularization of the affected parenchyma is observed, while the echogenically healthy-appearing regions exhibit normal or slightly increased vascularization (17). The vasculature improves along with the recovery. CAT may be associated with increased, normal, or decreased vascularization (12). Generally, thyroid scintiscan in SAT depicts decreased or no radionuclide uptake, while AT and CAT are characterized by only slightly reduced or focally changed iodine or technetium trapping. However, in case of atypical presentation, thyroid scintiscan might be of little value in differential diagnosis (10).

Though in most cases, clinical, biochemical and conventional US data are sufficient to provide an accurate diagnosis, in case of any doubts, sonoelastography may assist in differentiation of thyroiditis and help in treatment monitoring. In our study, sonoelastography was found to be effective in differentiating AT or SAT, both of which present significantly increased thyroid stiffness, and CAT characterized by only a slightly stiffer thyroid parenchyma, in comparison with that of a normal thyroid. According to our data, close to normal thyroid elastic properties in AT and SAT are restored with remission. Thus, the stiffness values were found to be useful in monitoring disease remission

Table 3 Thyroid tissue stiffness assessed qualitatively in a 4-point scale and the number of patients classified into the four groups.

\begin{tabular}{llllll}
\hline & $\begin{array}{l}\text { SAT } \\
\text { (baseline) }\end{array}$ & $\begin{array}{l}\text { SAT } \\
\text { (4-week follow-up) }\end{array}$ & $\begin{array}{l}\text { SAT } \\
(10-w e e k \text { follow-up) }\end{array}$ & CAT & CS \\
\hline Thyroid stiffness & & & & & \\
I & - & $4(22.2 \%)$ & $15(83.3 \%)$ & $6(33.3 \%)$ & $39(97.5 \%)$ \\
II & - & $13(72.2 \%)$ & $3(16.7 \%)$ & $12(66.7 \%)$ & $1(2.5 \%)$ \\
III & $1(5.6 \%)$ & $1(5.6 \%)$ & - & - & - \\
IV & $17(94.4 \%)$ & - & - & - & - \\
\hline
\end{tabular}

SAT, subacute thyroiditis; CAT, chronic autoimmune thyroiditis; CS, control subjects. 
A

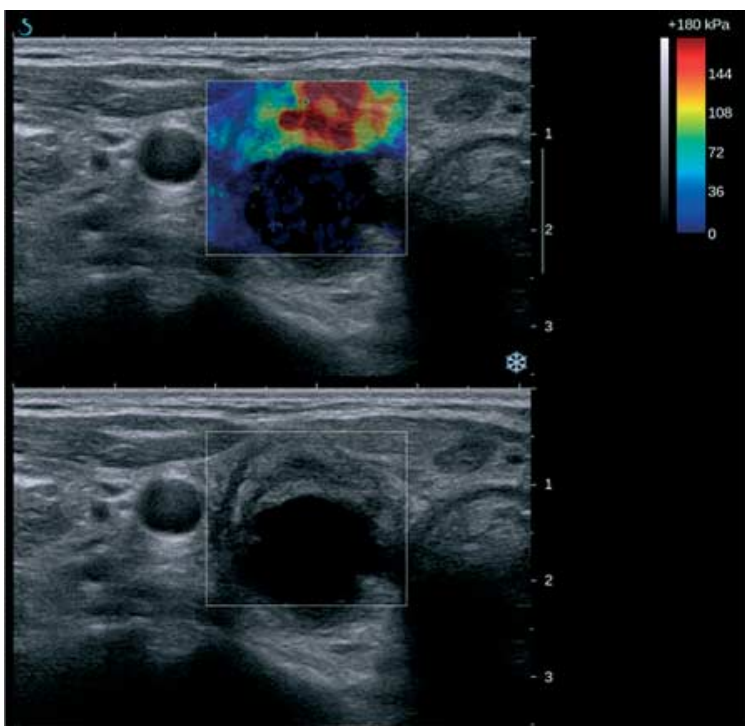

B

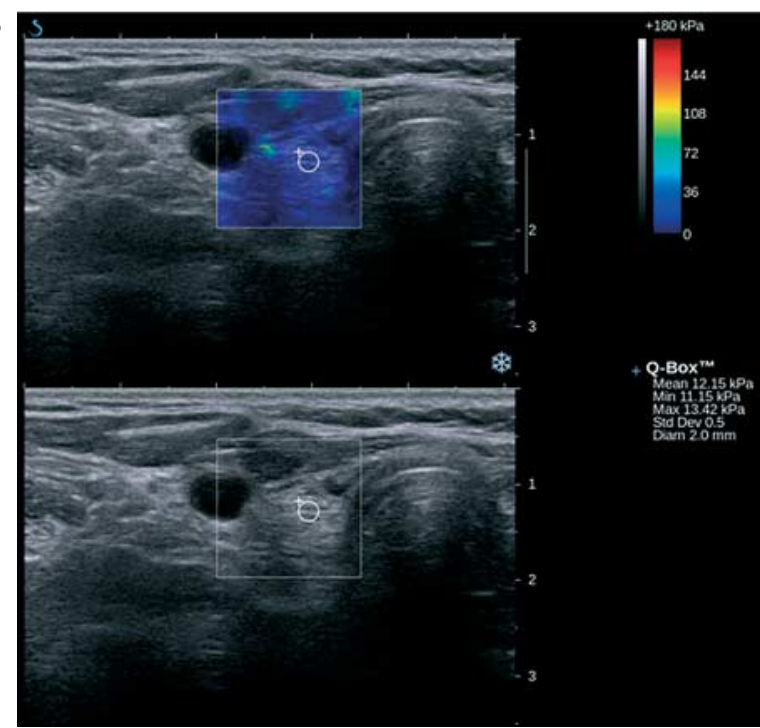

Figure 3 Thyroid stiffness in acute thyroiditis assessed (A) at baseline and $(B)$ during remission after treatment.

in AT and SAT. On the other hand, thyroid stiffness in CAT was not significantly influenced by the introduction of $\mathrm{L}_{-} \mathrm{T}_{4}$ treatment. The results of thyroid stiffness in our group of patients with CAT are comparable to those obtained in the study by Magri et al. (18), though a little higher. A slight discordance might be attributed to the different number of subjects included in these studies. Another important issue is that all patients diagnosed with CAT in our study were on $\mathrm{L}^{-} \mathrm{T}_{4}$ replacement therapy, while less than half of the patients enrolled in the study by Magri et al. (18) needed L-T $\mathrm{T}_{4}$. The mean value of elasticity in this subgroup of 14 patients was noted to be higher than that in patients with no thyroid dysfunction. Since the lymphocytic infiltration and fibrosis are connected with the extent of thyroid parenchymal changes and functional impairment, it may be assumed that the elasticity values in CAT may correlate with these two histological features. Moreover, thyroid stiffness in the recovery phase of SAT and in CAT was found to be very similar, which, during this phase, makes arriving at a differential diagnosis on the basis of sonoelastography quite difficult. What is worth mentioning is that the degree of tissue stiffness and localization of the regions characterized by disturbed elasticity in SAT corresponded with the localization and intensity of pain reported by the patients.

The dissimilarities of thyroid stiffness observed in the three types of thyroiditis may be attributed to the differences in histopathologic architecture (19). The cytological specimen in AT is mostly composed of neutrophils and amorphous components of pus. On the other hand, an early phase of SAT is characterized by the disruption of follicles and neutrophil infiltration, with subsequent aggregation of lymphocytes, histiocytes and plasma cells. Multinucleated giant cells are quite typical; however, polymorphonuclear and lymphocytic populations may also be present $(20,21)$. Consequently, the areas of injury are replaced by a chronic inflammatory infiltrate and fibrosis (21). CAT is characterized mainly by focal or diffuse infiltrate of small mononuclear lymphocytes and Hürthle cell changes (12). Nevertheless, there is a wide overlap in cytomorphological features of thyroiditis, and therefore cytological analysis may not alone be used to determine diagnosis (20).

Another important issue is the fact that changes in thyroid firmness induced by thyroiditis may affect the evaluation of stiffness of thyroid focal lesions. Some authors reported that the coexistence of Hashimoto's thyroiditis does not significantly influence the interpretation of the sonoelastographic image of concomitant thyroid nodules $(1,18,22)$. However, it is still to be evaluated whether other types of thyroiditis might influence the result of thyroid nodules' elasticity estimation. It is probable that SAT or AT, causing much more significant changes in elastic properties of the thyroid parenchyma than CAT, might importantly affect the estimated nodule firmness. As cases of coexistence of SAT with nodular goiter or thyroid cancer have been described, it seems best to postpone the assessment of the nodule stiffness until a complete recovery from SAT or AT (23).

In our study, the stiffness of thyroid parenchyma was assessed in both a qualitative and a quantitative manner and the obtained results were comparable. Recently, Park et al. (24) demonstrated a high interobserver variability of the qualitative methods of elastographic evaluation, where the elasticity image and score depended on the extent of tissue compression. On the other hand, since the technique of Shear Wave Elastography used in our study is devoid of the need for freehand external compression, it can be assumed to be 
operator independent, reproducible, and objective in the evaluation of tissue firmness (25).

The practical applicability of sonoelastography in thyroiditis might be limited, as several other clinical and laboratory tests coupled with conventional US usually provide a clinician with sufficient information to establish a diagnosis and decide on the management. Though palpation allows one to roughly assess the degree of thyroid firmness, elastographic imaging offers the quantitative and objective assessment of thyroid stiffness, an opportunity to follow changes in time, and the possibility to visualize the firmness of the dorsal parts of the thyroid. What is more, the examination is easy to perform and requires no more than 3-5 min in addition to the routinely performed conventional US (26).

Evidently, the changes of thyroid tissue stiffness observed in different types of thyroiditis are not specific and may not serve as the only factor determining the diagnosis. In addition to other factors, sonoelastography may be regarded as an adjunctive method supporting the diagnosis of thyroiditis, especially in cases of the so-called 'gray zone'. Furthermore, it allows physicians to monitor the recovery of patients from AT or SAT, and thus influences the titration of medications. It is interesting to note that elasticity in convalescents from SAT is still significantly higher than that in controls. However, it might be attributed to the relatively short observation period in our study. It is also possible that the elasticity would become completely normal over the time of 10 weeks or more. Additionally, the lack of complete restoration of normal thyroid stiffness might indicate the coexistence of CAT. Further studies on larger groups of patients are required to confirm whether persistently increased thyroid stiffness in the recovery phase of SAT might be indicative of long-term hypothyroidism or disease recurrence.

There were some important limitations in this study, which should be mentioned. First, the number of enrolled patients was relatively small. Secondly, the main objective of this research was to show general differences between sonoelastographic images of various types of thyroiditis. Hence, detailed description of sonoelastographic image variation and its correlation with biochemical and cytological findings for each of the particular conditions was not included. However, that could be an interesting idea for further exploration on a larger group of patients.

In conclusion, AT and SAT are associated with an increased stiffness of the thyroid parenchyma, which is restored with treatment to values close to normal. CAT is associated with only a minimal increase in the stiffness of thyroid tissue, which remains unchanged during therapy. Sonoelastography may assist in the diagnosis and treatment monitoring of AT, SAT and $\mathrm{CAT}$, as well as in the differentiation of the various types of thyroiditis.

\section{Declaration of interest}

The authors declare that there is no conflict of interest that could be perceived as prejudicing the impartiality of the research reported.

\section{Funding}

This study was supported by the State Committee for Scientific Research (grant nr N N402 545540 and 402/FNiTP/162/2009).

\section{References}

1 Gietka-Czernel M, Kochman M, Bujalska K, StachlewskaNasfeter E \& Zgliczynski W. Real-time ultrasound elastography a new tool for diagnosing thyroid nodules. Endokrynologia Polska $201061652-657$.

2 Lyshchik A, Higashi T, Asato R, Tanaka S, Ito J, Mai JJ, Pellot-Barakat C, Insana MF, Brill AB, Saga T, Hiraoka M \& Togashi K. Thyroid gland tumor diagnosis at US elastography. Radiology 2005237 202-211. (doi:10.1148/radiol.23630 41248)

3 Bojunga J, Herrmann E, Meyer G, Weber S, Zeuzem S \& FriedrichRust M. Real-time elastography for the differentiation of benign and malignant thyroid nodules: a meta-analysis. Thyroid $2010 \mathbf{2 0}$ 1145-1150. (doi:10.1089/thy.2010.0079)

4 Lippolis PV, Tognini S, Materazzi G, Polini A, Mancini R, Ambrosini CE, Dardano A, Basolo F, Seccia M, Miccoli P \& Monzani F. Is elastography actually useful in the presurgical selection of thyroid nodules with indeterminate cytology? Journal of Clinical Endocrinology and Metabolism 201196 E1826-E1830. (doi:10.1210/jc.2011-1021)

5 Rago $\mathrm{T} \&$ Vitti P. Role of thyroid ultrasound in the diagnostic evaluation of thyroid nodules. Best Practice $\mathcal{E}$ Research. Clinical Endocrinology \& Metabolism 200822 913-928. (doi:10.1016/ j.beem.2008.09.016)

6 Fatourechi MM, Hay ID, McIver B, Sebo TJ \& Fatourechi V. Invasive fibrous thyroiditis (Riedel thyroiditis): the Mayo Clinic experience, 1976-2008. Thyroid 201121 765-772. (doi:10.1089/thy.2010. 0453)

7 Slman R, Monpeyssen H, Desarnaud S, Haroche J, Fediaevsky Ldu P, Fabrice M, Seret-Begue D, Amoura Z, Aurengo A \& Leenhardt L. Ultrasound, elastography, and fluorodeoxyglucose positron emission tomography/computed tomography imaging in Riedel's thyroiditis: report of two cases. Thyroid $2011 \mathbf{2 1}$ 799-804. (doi:10.1089/thy.2010.0242)

8 Sporea I, Vlad M, Bota S, Sirli RL, Popescu A, Danila M, Sendroiu M \& Zosin I. Thyroid stiffness assessment by acoustic radiation force impulse elastography (ARFI). Ultraschall in der Medizin 201132 281-285. (doi:10.1055/s-00291246048)

9 Ruchala M, Szczepanek E \& Sowinski J. Sonoelastography in de Quervain thyroiditis. Journal of Clinical Endocrinology and Metabolism 201196 289-290. (doi:10.1210/jc.2010-1595)

10 Cases JA, Wenig BM, Silver CE \& Surks MI. Recurrent acute suppurative thyroiditis in an adult due to a fourth branchial pouch fistula. Journal of Clinical Endocrinology and Metabolism $2000 \mathbf{8 5}$ 953-956. (doi:10.1210/jc.85.3.953)

11 Nishihara E, Ohye H, Amino N, Takata K, Arishima T, Kudo T, Ito M, Kubota S, Fukata S \& Miyauchi A. Clinical characteristics of 852 patients with subacute thyroiditis before treatment. Internal Medicine 200847 725-729. (doi:10.2169/internalmedicine.47. 0740)

12 Onoda N, Kato Y, Seki T, Kurimoto M, Takano K, Ito Y \& Sato K. Increased thyroid blood flow in the hypoechoic lesions in patients with recurrent, painful Hashimoto's thyroiditis at the time of acute exacerbation. Endocrine Journal 200956 65-72. (doi:10.1507/ endocrj.K08E-194) 
13 Espinoza PG, Guendelman CL, Quevedo Limon LN \& Fernandez RJ. A comparison between two imaging techniques for the diagnosis of subacute thyroiditis (de Quervain thyroiditis): brief communication. Clinical Nuclear Medicine 201035 862-864. (doi:10. 1097/RLU.0b013e3181f49adc)

14 Fatourechi V, Aniszewski JP, Fatourechi GZ, Atkinson EJ \& Jacobsen SJ. Clinical features and outcome of subacute thyroiditis in an incidence cohort: Olmsted County, Minnesota, study. Journal of Clinical Endocrinology and Metabolism $2003 \mathbf{8 8}$ 2100-2105. (doi:10.1210/jc.2002-021799)

15 Bennedbaek FN \& Hegedus L. The value of ultrasonography in the diagnosis and follow-up of subacute thyroiditis. Thyroid $1997 \mathbf{7}$ 45-50. (doi:10.1089/thy.1997.7.45)

16 Park SY, Kim EK, Kim MJ, Kim BM, Oh KK, Hong SW \& Park CS. Ultrasonographic characteristics of subacute granulomatous thyroiditis. Korean Journal of Radiology $2006 \quad 7$ 229-234. (doi:10.3348/kjr.2006.7.4.229)

17 Kunz A, Blank W \& Braun B. De Quervain's subacute thyroiditis colour Doppler sonography findings. Ultraschall in der Medizin 200526 102-106. (doi:10.1055/s-2005-858120)

18 Magri F, Chytiris S, Capelli V, Alessi S, Nalon E, Rotondi M, Cassibba S, Calliada F \& Chiovato L. Shear wave elastography in the diagnosis of thyroid nodules: feasibility in the case of coexistent chronic autoimmune Hashimoto's thyroiditis. Clinical Endocrinology 2012 76 137-141. (doi:10.1111/j.1365-2265.2011.04170.x)

19 Varghese T. Quasi-static ultrasound elastography. Ultrasound Clinics 20094 323-338. (doi:10.1016/j.cult.2009.10.009)

20 Jayaram G, Marwaha RK, Gupta RK \& Sharma SK. Cytomorphologic aspects of thyroiditis. A study of 51 cases with functional, immunologic and ultrasonographic data. Acta Cytologica 198731 $687-693$.
21 Kabalak T \& Ozgen AG. Familial occurrence of subacute thyroiditis. Endocrine Journal 200249 207-209. (doi:10.1507/ endocrj.49.207)

22 Asteria C, Giovanardi A, Pizzocaro A, Cozzaglio L, Morabito A, Somalvico F \& Zoppo A. US-elastography in the differential diagnosis of benign and malignant thyroid nodules. Thyroid 2008 18 523-531. (doi:10.1089/thy.2007.0323)

23 Nishihara E, Hirokawa M, Ohye H, Ito M, Kubota S, Fukata S, Amino N \& Miyauchi A. Papillary carcinoma obscured by complication with subacute thyroiditis: sequential ultrasonographic and histopathological findings in five cases. Thyroid 200818 1221-1225. (doi:10.1089/thy.2008.0096)

24 Park SH, Kim SJ, Kim EK, Kim MJ, Son EJ \& Kwak JY. Interobserver agreement in assessing the sonographic and elastographic features of malignant thyroid nodules. AJR. American Journal of Roentgenology 2009193 W416-W423. (doi:10.2214/AJR.09. 2541)

25 Sebag F, Vaillant-Lombard J, Berbis J, Griset V, Henry JF, Petit P \& Oliver C. Shear wave elastography: a new ultrasound imaging mode for the differential diagnosis of benign and malignant thyroid nodules. Journal of Clinical Endocrinology and Metabolism 201095 5281-5288. (doi:10.1210/jc.2010-0766)

26 Rago T, Santini F, Scutari M, Pinchera A \& Vitti P. Elastography: new developments in ultrasound for predicting malignancy in thyroid nodules. Journal of Clinical Endocrinology and Metabolism 200792 2917-2922. (doi:10.1210/jc.2007-0641)

Received 21 August 2011

Revised version received 11 November 2011

Accepted 5 December 2011 